\title{
Multimedia Goes Corporate
}

\section{Patrick Schmitz Ludicrum Enterprises}

A lthough many people may think of consumer multimedia as more exciting or cooler than corporate multimedia, the luxury of a more powerful and up-to-date platform often means the opposite is true. Many IT managers mandate a consistent desktop configuration, where the public/consumer Web is a morass of platforms and versions. In addition, although corporate multimedia is essentially utilitarian, it still must employ good design, pleasing aesthetics, and a certain degree of excitement. The same folks who are corporate users by day are Web and television consumers in the evening. Sensitized by consumer media, corporate users will be bored or put off by poorly produced or flat corporate content. Effective corporate multimedia must be compelling, cost-effective, and productive. As a result, some great content and content models have emerged that merit attention from the research community-there is much to learn from both the successes and failures.

\section{Content versus conferencing}

I distinguish between multimedia as content and multimedia for conferencing. Conferencing uses media for live communication (spatial telepresence but no temporal telepresence-a glorified telephone) whereas content uses media in authored or archived communication (including narrative and/or interactive content and often distinguished by temporal telepresence). The conferencing domain shares some problems and challenges with multimedia content, but the main challenges are different, and solutions for the conferencing domain often don't help with core problems in archived or authored multimedia. For example, video telephony has different goals and constraints than on-demand network video, and the codecs and transport protocols aren't generally interchangeable between the domains.

A number of significant problems are common to both domains, however. The big three are production, capture and synchronization, and infrastructure:

I At the top of the list is the difficulty of recording good quality video and audio. Setting up lighting, cameras, and microphones (still) requires skilled professionals and is a major impediment for many organizations.

- A related problem for lectures, classes, speeches, and so forth is capturing and synchronizing associated media such as presentation slides, whiteboards, and audience/participant feedback and questions.

- Finally, the infrastructure costs are considerable for dedicated spaces (such as lecture halls configured for capture and telemeeting rooms) or for the alternative of additional equipment for all employees (such as cameras, lighting, and microphones in each office).

Another obvious infrastructure problem is end-to-end network capacity, and for a long time, this was more severe. However, this gets my vote for the problem "Most Likely To Be Solved."

Content and conferencing domains both have the potential to increase and decrease employee productivity:

I Productivity gains. Telelearning, telepresentation, and telemeetings promise cost savings compared to travel and the dedicated time away from regular duties. However, these gains are only realized if telepresence factors are well addressed, including immersion and especially feedback for live, spatial telepresence. For temporal telepresence, control, navigation support, and easy accessibility are crucial.

- Productivity losses. Attendance becomes easier, so people might participate when they shouldn't. Security protocols might be harder 
to implement for telesolutions than for simple badge-at-the-door physical solutions. Abuse of nonproductive material (entertainment and worse) might decrease productivity and has been a significant concern for management.

Although content and conferencing share general challenges, the implications have played out differently in the two domains. Oversimplifying a bit, conferencing applications haven't seen much success, where at least some content applications have seen wide adoption. To understand why, we need to look at individual use cases.

\section{Sometimes you lose ...}

A number of widely hyped multimedia applications haven't worked well or have failed to gain wide acceptance.

\section{High-immersion telemeetings}

This includes cases that traditionally need considerable social interchange, such as business negotiations, customer meetings for important sales, and so forth. The problem is that social telepresence issues remain unsatisfied; we are too used to things like shaking hands and sharing lunch or drinks, and we still rely heavily on subtle factors like smell and physical proximity. Production issues are difficult as always, and the economic trade-offs of the cost of dedicated rooms and networks versus the cost of travel are underwhelming.

In cases where social contact isn't as important (such as internal meetings or discussions), telemeeting with video links isn't effective enough to justify the expense and logistical complexity over a telephone conference call.

\section{Telecommuting}

The technology is largely in place, with inexpensive PCs in most homes and home offices, good network connectivity, and virtual private network (VPN) support widely available. There are also economic incentives, both in terms of reduced facilities costs in corporate offices and federal tax incentives for telecommuting to reduce traffic congestion. Surveys consistently show a high interest among workers in telecommuting. Proponents note that many companies (some say more than two-thirds of Fortune 500 companies) have telecommuting programs in place and quote figures that as many as one in five employees telework once a week or more. ${ }^{1}$ However, other surveys of human-resources managers indicate the numbers are lower and are decreasing; some surveys show employers are much less friendly to telework than the hype seems to indicate, especially as the economy has slowed and eased a tight office space market. ${ }^{2,3}$

Ultimately, other than for a few jobs like journalism (the source of much hype on the subject) and telephone-based support services, most people need to be physically in an office setting or face to face with coworkers, collaborators, or customers to be effective. Although telecommuting can in theory be effective for many knowledge workers, it requires considerable adjustment to management and workplace logistics. Managers need to rework their management style to ensure sufficient communication, and the rest of an organization must change many habits to effectively accommodate a telecommuting peer. Few companies truly support telecommuting (official policies notwithstanding), even in technology centers like Silicon Valley or the Pacific Northwest. In an ironic but representative personal example from my time at Microsoft Research, managers strongly discouraged telecommuting (even for multimedia and telepresence researchers).

The point isn't that technology for telecommuting isn't adequate (it is), but rather that no amount of technology can force social change.

\section{Visual entertainment and infotainment}

Some examples of this are streaming video for news, entertainment, and early incarnations of push models such as PointCast. Note that this isn't really corporate media in the sense of being produced or authored within the organization but rather in the sense that it is (or was) readily available to and consumed by corporate Web users. As such, it was the focus of much concern in IT departments and management offices.

News and entertainment video creates numerous significant problems in the work environment, especially in contrast with network radio. The central issue is one of ambience. Ambient media can surround the end user without being the central focus and can be effective or useful in the background. The content of Internet news and entertainment video generally isn't ambient. This means that the user's attention must be focused on the media to the exclusion of any other tasks. Not surprisingly, the effect of this (and the prevailing fear of managers) is to reduce productivity. Some news may provide an offsetting gain in business knowledge, but the use of rich media in this context generally doesn't provide incremen- 


\section{The rapidly accumulating} body of telelearning and

\section{telepresentation content will}

\section{be invaluable to researchers}

studying issues of

representation, retrieval, and user-centered presentation.

tal value over simple text and graphics.

Another issue, particularly when network bandwidth is more constrained (especially on the connection to the external network), is that video and even the graphics-heavy content of some push channels can add a significant load on a network, particularly when multiplied over many individual users. If the content isn't clearly supporting productive work (as with telelearning), IT managers and network administrators find it too tempting to just cut off the offending material-for example, by blocking required ports and protocols. Strict policies restricting nonwork-related Web use have become common, at least partly in response to the perceived abuse of media-rich content. ${ }^{4}$

\section{Content reuse and repurposing}

It should be possible for office workers to easily incorporate existing multimedia assets in presentations, memos, and so on. At this point, this kind of application is still rare. The reasons are numerous:

- Commonly available authoring tools don't make this easy for users.

- Existing and widely deployed media server architectures don't support the needed reference capabilities-especially fragment references from within common document types.

I Discovery is too hard. Would-be authors simply don't know about existing content assets. A major reason for this is the lack of annotation. Currently annotation is too time consuming, and automatic annotation software is far too primitive to provide the necessary annotation information for things like video and audio.

The last issue poses a significant problem for multimedia on the semantic Web. Where semantic extraction from text-based documents and databases is advancing in leaps and bounds, semantic extraction from still images is still poor, and semantic extraction from video remains a pipe dream. Semantic extraction from associated audio has somewhat more promise, but it still requires video synchronized to audio containing speech, where the speech audio can be sufficiently separated from any other audio to enable speech recognition with high reliability. Although this at least seems like a tractable problem, it's likely to require too much manual intervention to be useful in practice for quite some time.

\section{And sometimes you win ...}

So is the story of multimedia in corporations all gloom? Not at all. In fact, some content models and applications are providing real success stories.

\section{Corporate telelearning and telepresentation}

The use of live, archived, and on-demand courses, lectures, presentations, and informal seminars has become fairly widespread in corporations. This is a genuine success story in applied multimedia, which illustrates a good balance of costs and benefits. The highlights include the following:

I Content production requires a moderate investment in the physical setting, in recording equipment, a minimal training of teachers and speakers, and a production engineer. The logistics of recording events has minimal impact on the event itself, and the logistics of supporting the media server and content can often piggyback on existing intranet services. The content requires little client setup or management because companies can easily target it to popular browsers such as Microsoft Internet Explorer or RealNetworks players.

I The most significant benefit is exposure of a wider range of employees to lectures, classes, and other types of training material. The spatial and temporal telepresence is important to employees with busy schedules and deadlines, not to mention the advantages for a geographically distributed workforce.

I Because there is a strong speech component to 
this content, it can be somewhat ambient. Employees can listen in on lectures of marginal interest while continuing to do simple tasks like scanning email or eating lunch. I can't count the number of research lectures I "browse" in this manner-if and when they get interesting I can focus on the presentation, but I don't have to commit an hour or two away from my office to take a chance on a given lecture.

I The technology lets client users pause and resume, seek ahead to specific points, and replay critical segments. This client-side control is a significant benefit to learning as well as to scheduling flexibility (for example, accommodating interruptions). This functionality has the unfortunate side effect that scaling with multicast isn't an option.

As noted earlier, what isn't yet widely available is a mechanism to easily annotate and reuse this kind of media-for example, to excerpt a lecture or some training material, add comments, and pass it on to peers for more directed viewing. Structured description, reference, and annotation formats are in place (such as the Synchronized Multimedia Integration Language, XPointer/ XPath, and Resource Description Framework), but the integrated application for this class of use cases is still in development.

\section{Network radio}

Internet radio has succeeded for numerous reasons, all of which illuminate how media is used and abused in corporate environments:

I Because radio content is mostly ambient, it doesn't require workers' focus and so doesn't (or isn't perceived to) impede their productivity. On the contrary, because music can improve their mood, it may help productivity.

I Because the required bandwidth is relatively low, it puts little drain on network resourcescertainly much less than video.

I Because conventional radios often get poor reception inside office buildings, network radio solves a problem for people who want to listen to the radio at work. By the same token, network radio extends the reach of conventional radio, so it's attractive to broadcasters. Because little infrastructure is required to repurpose the broadcast content, it's fairly simple for broad- casters to support a network mirror (or perhaps more appropriately a network "echo").

I Finally, because network radio is potentially more interactive than traditional radio (for example, leveraging HTML content with hyperlinks delivered in sync with advertisements), and because the demographics of network radio listeners is attractive, the new medium is appealing to advertisers. A downside for local advertisers is that many network listeners may be geographically remote from the broadcast origin.

Internet radio is a great case study in the business and economics of Internet multimedia. It's also a good illustration of how commercial multimedia can fit into a corporate environment.

\section{Learn from the corporate experience}

The world of corporate multimedia is in many respects far ahead of the broader Web environment, leveraging consistent and up-to-date client platforms and high-bandwidth local area network connectivity to deploy compelling multimedia applications that enhance productivity. The success stories are encouraging, while the failures remind us that social and logistical issues can greatly impede acceptance of technology. The rapidly accumulating body of telelearning and telepresentation content will be invaluable to researchers studying issues of representation, retrieval, and user-centered presentation, not to mention semantic extraction and association for multimedia content.

MM

\section{References}

1. D. Davis and K. Polonko, Telework in the United States: Telework America Survey 2001, Int'I Telework Association and Council, Wakefield, Mass., 2001.

2. S. Dubowski, "Techies Want Tools Instead of Tap Dancing Lessons," Itrecruitermag (http://www. itrecruitermag.com), 8 Nov. 2000.

3. B. Harris, "Companies Turning Cool to Telecommuting Trend," Los Angeles Times, 28 Dec. 2000, Record Edition, p. A1.

4. The Year 2001 Corporate Web and Email Usage Study, Elron Software, Burlington, Mass., 2001, http:// www.elronsoftware.com/pdf/NFOreport.pdf.

Contact Patrick Schmitz at cogit@ludicrum.org. Contact Media Impact editor Frank Nack at CWI, Kruislaan 413, PO Box 94079, 1090 GB Amsterdam, The Netherlands, email Frank.Nack@cwi.nl. 